\title{
Cribado de problemas psicosociales en Atención Primaria
}

\author{
E. Fabregat Ferrer, MJ. Palomares Gimeno \\ Pediatras. CS Gran Vía. Castellón. España.
}

Rev Pediatr Aten Primaria. 2009; I : 425-37

Elena Fabregat Ferrer, fabregat_ele@gva.es

\begin{abstract}
Resumen
Los problemas psicosociales son muy prevalentes en la infancia y los pediatras de Atención Primaria debemos mejorar nuestra capacidad para detectarlos. La orientación y diagnóstico precoz reducen la morbilidad y mejoran el pronóstico; por eso proponemos incorporar una herramienta de cribado en el Programa de Salud Infantil. Hemos utilizado un cuestionario validado en la población española "Strengths and Difficulties Questionnaire" (SDQ) fácil de usar y con capacidad demostrada en Atención Primaria.

Presentamos nuestra experiencia en un centro de salud tras realizar el SDQ -versión padres-en el control de salud de los 5-6 años durante un período de 2 años.

Resultados: detectamos un 15\% de niños con dificultades. Los problemas de conducta han sido los más frecuentes a esta edad. Factores de vulnerabilidad como la patología psiquiátrica en los padres y la monoparentalidad son predisponentes para presentar problemas emocionales y en las relaciones con los compañeros. Los niños inmigrantes tienen mayor incidencia de problemas de relación con iguales. La incorporación de otros informadores, como el profesor, mejoraría el rendimiento del cribado.

Conclusión: el SDQ-padres es un instrumento útil en Atención Primaria para el cribado de problemas psicosociales en la infancia, bien aceptado por las familias y fácil de interpretar para los profesionales. Se precisan más estudios para desarrollar un algoritmo que ayude a decidir cuándo solicitar información a los servicios educativos y mejorar así nuestra capacidad diagnóstica.
\end{abstract}

Palabras clave: Cribado, Psicosocial, Atención Primaria, Infancia, Salud mental.

\section{Abstract}

Psychosocial problems are prevalent in childhood and primary care pediatricians should improve our ability to detect them. Orientation and early diagnosis reduce morbidity and improve prognosis. For this reason we propose the introduction of a screening tool in the children's health programme. We have used a questionnaire validated with Spanish population: the Strengths and Difficulties Questionnaire (SDQ). It is easy to use and it has proved to be efficient in primary care.

Las autoras declaran no presentar conflictos de intereses en relación con la preparación y publicación de este artículo. 
We present our experience applying the $S D Q$, parent version, in a primary care centre. It has been applied in the 5-6 years old children health control for two years.

Results: we have identified $15 \%$ of the children presenting some difficulties. The most common problems found at this age are behavioural problems. Parent's psychiatric conditions and monoparentality are predisposing factors that can lead to emotional and relation problems. Immigrant children present a high incidence in relation problems with their equals. The incorporation of other information sources such as the teachers, could improve the screening performance.

Conclusion: the parent-SDQ is a useful tool in primary care for the screening of childhood psychosocial problems. It is well accepted by the families and it is easily interpreted by the professionals. More studies are necessary to develop an algorithm that can help professionals to decide when it is necessary to ask for information to the Educative Services in order to improve our diagnosis capacity.

Key words: Screening, Psychosocial, Primary Care, Children, Mental health.

\section{Introducción}

Los pediatras hemos asistido desde nuestra posición de observadores privilegiados del desarrollo del niño y de su familia, a un cambio secular en el modo de crianza de los hijos, los hábitos disciplinarios y las costumbres sociales ${ }^{1}$. La sociedad se ha transformado y estos cambios han modificado los patrones de relación familiar. Las redes sociales y familiares de apoyo son escasas, la familia extensa casi ha desaparecido con la dispersión geográfica que impone el mercado laboral y las familias monoparentales son cada vez más frecuentes (sea por separación conyugal, madres solteras, fallecimiento, abandono, adopción por personas solteras, etc.).

La precariedad laboral es común entre la población autóctona joven y aún más en el inmigrante que ha pasado de un $2 \%$ en los años noventa a representar en la actualidad un 15\% de la población pediátrica en nuestro medio ${ }^{2}$.

La cultura de nuestro tiempo está vertebrada por los medios de comunicación y muy especialmente por la televisión. Los valores dominantes priman la vistosidad, rapidez y el refuerzo positivo inmediato nos llega a través de la publicidad, dibujos animados, videojuegos, etc. Cada vez hay menos oportunidades para las estrategias reflexivas y la atención sostenida. Los modelos que ofrece este medio son muchas veces inadecuados y no cabe duda de que, después de la familia, son la influencia más importante en el desarrollo de nuestros hijos s.4 $^{3,4}$.

Los problemas psicosociales se han convertido en una causa muy frecuente de consulta en Pediatría de Atención Primaria: alteraciones del sueño, del desarrollo psicomotor, llanto, problemas de comportamiento, fracaso escolar, crisis 
del adolescente, enuresis, encopresis, anorexia, etc.; de hecho, casi la mitad de los motivos de consulta se relacionan con aspectos educacionales, psicosociales o de conducta 5 .

Se estima que 1 de cada 5 niños tendrá a lo largo de su infancia o adolescencia algún trastorno de salud mental. La bibliografía internacional ofrece valores del $5-25 \%^{6}$. Ese intervalo tan amplio puede explicarse por los diferentes diseños de los estudios según su objetivo, selección de la muestra, diferentes criterios diagnósticos utilizados, etc., pero la cifra más repetida es $15-20 \%{ }^{6}$. Los estudios españoles aportan cifras similares $^{7-10}$.

La capacidad de detección en Atención Primaria (AP) alcanza solo un $2 \%$ de niños y adolescentes con morbilidad psicopatológica ${ }^{10}$. Sabemos que la precocidad en la identificación y diagnóstico de las enfermedades mentales permite un manejo adecuado y mejora el pronóstico y rendimiento terapéutico. El reto que afrontamos los pediatras de $\mathrm{AP}^{11,12}$ es reconocer a estos niños para poner a su alcance el tratamiento más oportuno. Nos enfrentamos a múltiples dificultades: el tiempo limitado en las consultas, el déficit en formación específica en Psicopatología infantil y del desarrollo, la falta de entrenamiento en el uso de instrumentos de cribado, escasas posibilidades de colaborar con Salud Mental Infantil, circunstancias que hacen que nos sintamos incómodos cuando se plantea un problema de este tipo y nos inhibamos ${ }^{13}$.

Decidimos buscar un cuestionario validado y corto que permitiera identificar a los niños con problemas emocionales o de comportamiento y mejorar nuestra capacidad de detección. El control del Programa de Salud Infantil de los 5-6 años era un buen momento ${ }^{14,15}$, debido a que las herramientas de cribado están validadas a esta edad, el niño está escolarizado y ha alcanzado una cierta madurez psicoafectiva. El Strengths and Difficulties Questionnarie (SDQ) era una opción prometedora; desarrollado por Robert Goodman en el Instituto de Psiquiatría de Londres en $1997^{16}$. Es el instrumento más ampliamente utilizado en investigación en Salud Mental Infantil17. $\mathrm{Ha}$ demostrado sus propiedades psicométricas en diferentes culturas con diferentes puntos de corte ${ }^{18-23}$. En España la primera validación la ha realizado el equipo de Rodríguez Hernández en Canarias ${ }^{9,24,25}$, y lo más importante para nosotros, ha demostrado su utilidad cuando se ha aplicado en $\mathrm{AP}^{26}$.

Descartamos el CBCL (Child Behavioral Checklist) por su extensión (150 ítems) o Conners (de 10-90 ítems) por recoger 
solo problemas de hiperactividad y conducta. El Cuestionario de Capacidades y Dificultades (SDQ) abarca un amplio espectro de problemas psicosociales y es muy bien aceptado por la población por su lenguaje accesible, brevedad y por incluir datos positivos sobre el niño.

Utilizamos el SDQ-castellano/versión padres, que puede descargarse de forma gratuita de la página web www.sdqin fo.com. Está traducido a más de 40 idiomas entre ellos castellano, catalán, gallego, euskera y rumano.

Los objetivos son: incorporar un cuestionario validado al Programa de Salud Infantil a los 5-6 años que ayude a identificar los problemas psicosociales de mayor prevalencia en la infancia; conocer los problemas de nuestra población y presentar los resultados obtenidos.

\section{Material y métodos}

Se realizó un estudio descriptivo analítico transversal a los niños que acudieron a la consulta del centro de salud para el control de salud de los 5-6 años desde febrero de 2007 a febrero de 2009.

Se pidió la cumplimentación voluntaria del test SDQ en la consulta al padre o madre acompañante (mayoritariamente madres). A los niños acompañados por un cuidador distinto se les entregó el cuestionario para devolver en una visita posterior. A los padres con escaso dominio del castellano se les facilitó la versión del SDQ traducida a su idioma.

El cuestionario consta de 25 atributos psicológicos divididos en 5 escalas: síntomas emocionales, problemas de conducta, síntomas de hiperactividad/falta de atención, problemas con los compañeros y conducta prosocial. Cada escala incluye 5 ítems que se puntúan 0, 1 ó 2 (nunca, algunas veces o a menudo) cada uno, obteniéndose un valor en un rango 0-10 para cada escala. El total de dificultades (TD) se obtiene de la suma de los 20 ítems de dificultades (rango de 0-40).

Tras la corrección de los cuestionarios por los pediatras, los resultados se volcaron en la visita programada de los 5-6 años como información relevante del perfil psicológico del niño y se comunicaron a los padres en su siguiente visita a demanda.

Se revisó la historia clínica informatizada de los padres, disponible en nuestro centro de salud desde 2003 (últimos 5 años) registrando la edad actual de los padres y los diagnósticos relacionados con patología psiquiátrica o abuso de sustancias.

El Centro de Salud Gran Vía está ubicado en la periferia de la ciudad de Castellón próximo a la antigua N-340. Da co- 
bertura a una población bastante joven de clase media trabajadora, es una zona de expansión con una tasa de natalidad elevada (20\% niños menores de 2 años) y con un $15 \%$ de población inmigrante.

El total de niños de 5-6 años asignados a nuestro centro de febrero de 2007 a febrero de 2009 fueron 257 niños.

Finalmente se recogió en una base de datos Excel $^{\circledast}$ los siguientes parámetros de cada niño: puntuación del cuestionario SDQ en sus diferentes escalas y un sexto parámetro, el total de dificultades (TD) obtenido de la suma de las 4 escalas de dificultades; además sexo, país de origen, circunstancias familiares especiales (monoparentalidad) y la edad y patología psiquiátrica de los padres.

\section{Resultados}

Se obtuvieron 179 cuestionarios SDQ correctamente cumplimentados, que corresponde al $70 \%$ de la población diana (257 niños). Del 30\% restante: un 14\% no acude al centro de salud habitualmente y el resto no devolvieron el cuestionario o lo hicieron de forma incompleta. Solo en un caso los padres se negaron a realizarlo.

De los 179 niños un $57,5 \%$ son varones y $42,5 \%$ son mujeres.

El $83,8 \%$ son familias españolas y el $16,2 \%$ son familias procedentes de otros países, la mayoría de Rumania, seguido de Colombia, Nigeria, Marruecos, Argentina, Venezuela, China, Argelia y Macedonia.

El $11,7 \%$ de los niños conviven habitualmente con un solo progenitor (familia monoparental). Dato que puede tener sesgo porque no siempre esta información está reflejada en la historia. Posiblemente el porcentaje sea mayor.

Un $31 \%$ de niños tienen un padre o madre diagnosticado de patología psiquiátrica (55 padres o madres) por su médico de familia. El diagnóstico más frecuente es ansiedad, seguido de depresión. El abuso de alcohol es la adicción referida en 6 casos.

La media de edad de las madres es de 36 años y la de los padres de 39 años.

Los puntos de corte para la interpretación de los resultados de los cuestionarios se encuentran reflejados en la tabla I, tanto los originales de Goodman ${ }^{16}$, disponibles en www.sdqinfo.com, como los de Rodríguez ${ }^{9,24,25}$ para población española. Los resultados obtenidos en el test SDQ (tabla II) según los puntos de corte validados para población española muestran que nuestros niños de 5-6 años presentan los siguientes problemas por orden de frecuencia: $10,6 \%$ problemas de conducta, $8,4 \%$ de relaciones con compañeros, 4,5\% emocionales, 
Tabla I. Interpretación de resultados. Puntos de corte del test SDQ-versión padres, para niños de 4-16 años

\begin{tabular}{|c|c|c|c|c|c|c|}
\hline & \multicolumn{2}{|c|}{ Normal } & \multicolumn{2}{|c|}{ Límite } & \multicolumn{2}{|c|}{ Anormal } \\
\hline & Goodman & Rodríguez & Goodman & Rodríguez & Goodman & Rodríguez \\
\hline Síntomas emocionales & $0-3$ & $0-4$ & 4 & 5 & $5-10$ & $6-10$ \\
\hline Problemas de conducta & $0-2$ & $0-3$ & 3 & 4 & $4-10$ & $5-10$ \\
\hline Hiperactividad & $0-5$ & $0-6$ & 6 & $7-8$ & $7-10$ & $9-10$ \\
\hline Problemas de relación & & & & & & \\
\hline con compañeros & $0-2$ & $0-3$ & 3 & 4 & $4-10$ & $5-10$ \\
\hline Total dificultades (TD) & $0-13$ & $0-16$ & $14-16$ & $17-19$ & $17-40$ & $20-40$ \\
\hline Prosocial & $6-10$ & $6-10$ & 5 & 5 & $0-4$ & $0-4$ \\
\hline
\end{tabular}

Tabla II. Resultados del test SDQ en niños de 5-6 años. Número absoluto de pacientes y porcentajes

\begin{tabular}{lcccc}
\hline & Autor & Normal & Límite & Anormal \\
\hline Problemas emocionales & Goodman & $145(81,05 \%)$ & $16(8,9 \%)$ & $18(10,05 \%)$ \\
& Rodríguez & $161(89,9 \%)$ & $10(5,6 \%)$ & $8(4,5 \%)$ \\
\hline Problemas de conducta & Goodman & $114(67,7 \%)$ & $28(15,6 \%)$ & $37(20,7 \%)$ \\
& Rodríguez & $142(79,3 \%)$ & $18(10,1 \%)$ & $19(10,6 \%)$ \\
\hline Problemas de hiperactividad & Goodman & $151(73,2 \%)$ & $21(11,7 \%)$ & $27(15,1 \%)$ \\
& Rodríguez & $132(84,9 \%)$ & $20(11,2 \%)$ & $7(3,9 \%)$ \\
\hline Problemas con compañeros & Goodman & $130(72,8 \%)$ & $19(10,6 \%)$ & $30(16,6 \%)$ \\
& Rodríguez & $149(83,6 \%)$ & $15(8,4 \%)$ & $15(8,4 \%)$ \\
\hline Total dificultades (TD) & Goodman & $131(73,2 \%)$ & $20(11,2 \%)$ & $28(15,6 \%)$ \\
& Rodríguez & $151(84,35 \%)$ & $18(10,05 \%)$ & $10(5,6 \%)$ \\
\hline Prosocial & Ambos & $166(92,75 \%)$ & $7(3,9 \%)$ & $6(3,35 \%)$ \\
\hline Fuentes: Goodman $^{16}$, Rodríguez $^{24}$. & & & & \\
\hline
\end{tabular}

3,9\% de hiperactividad/falta de atención. Según los puntos de corte de Goodman ${ }^{16}$ la prevalencia de problemas es mayor aunque las proporciones se mantienen (figura 1). El parámetro prosocial valora las habilidades sociales de los niños y en nuestra población se observa que el 3,3\% presenta dificultades en este sentido.
La puntuación del parámetro total dificultades (TD) es representativo del conjunto de dificultades. En nuestro medio (figura 2) el 5,6\% de los niños de 56 años presentan dificultades o problemas según el test SDQ y un $10 \%$ puntúa en el límite.

En la tabla III aparecen las puntuaciones medias de cada parámetro del cues- 
Figura 1. Porcentaje de pacientes con resultados anormales en el test SDQ según puntos de corte de Goodman ${ }^{16}$ y Rodríguez ${ }^{24}$.

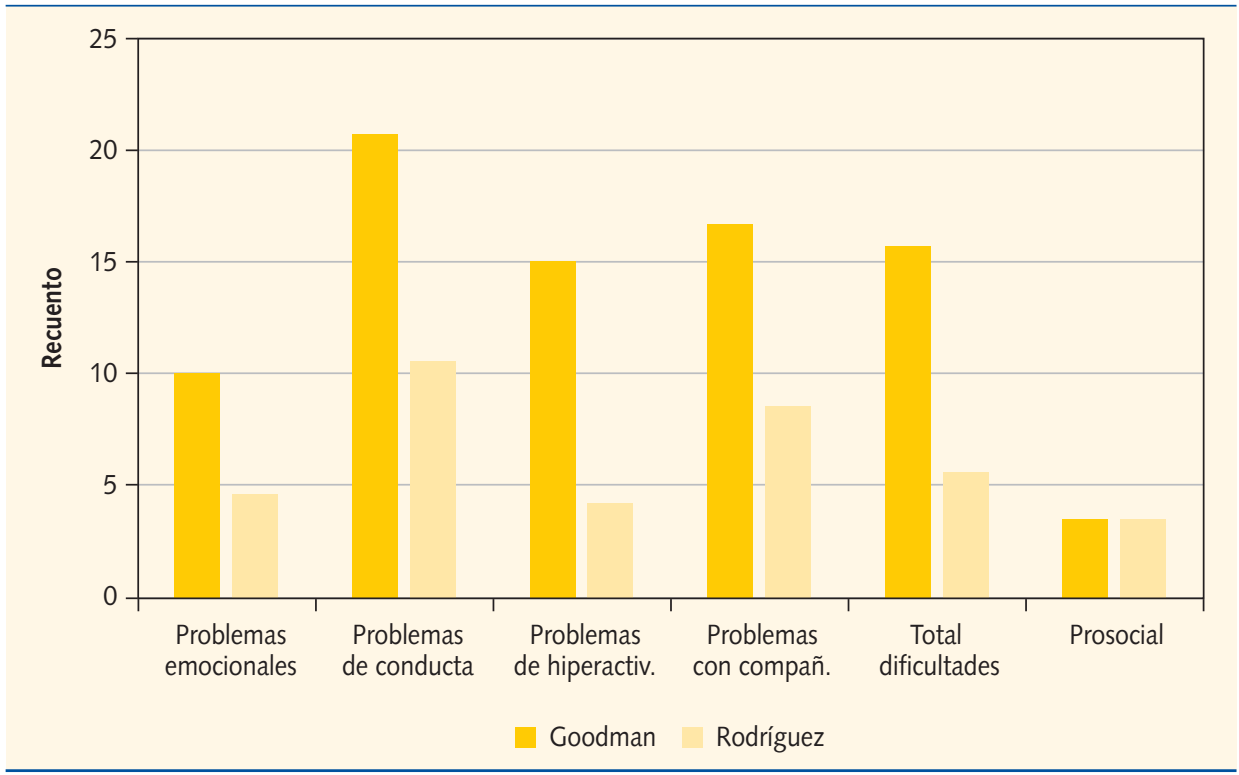

Figura 2. Total dificultades (TD): porcentajes de pacientes según puntos de corte españoles (Rodríguez ${ }^{24}$ ).

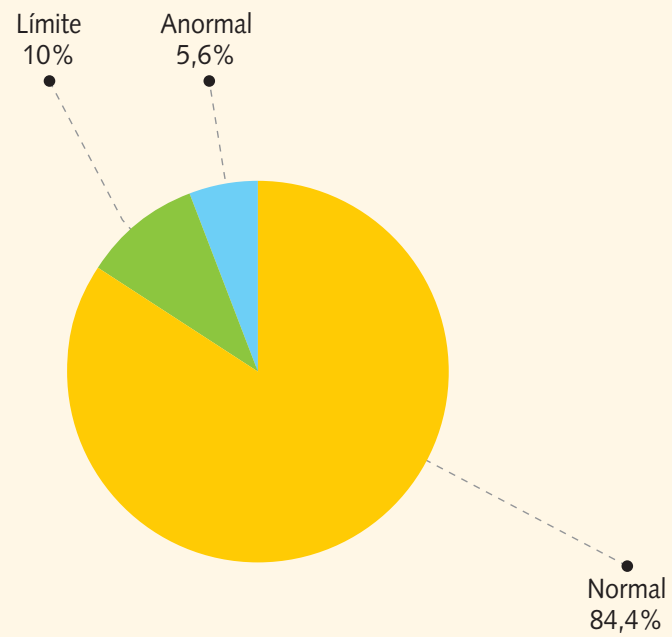




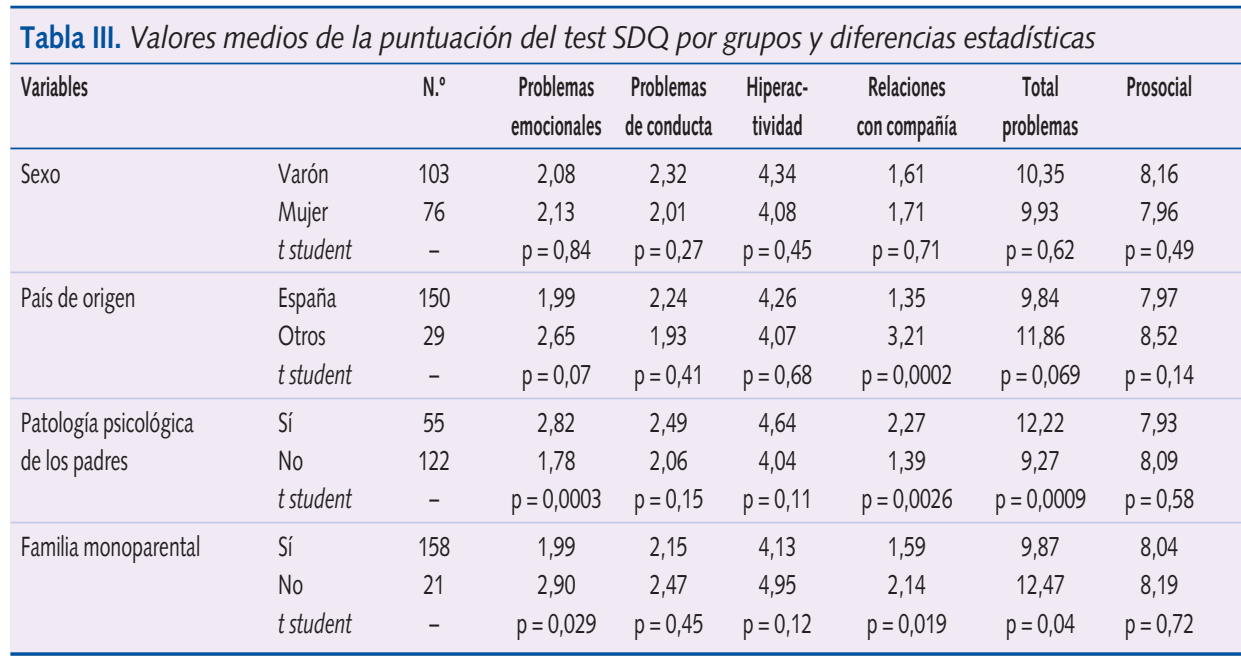

tionario separadas por sexo y los factores de vulnerabilidad estudiados. Para comparar los valores medios se aplicó la prueba $t$ de student en cada uno de los grupos, observando los siguientes resultados. El sexo no es un factor determinante; no hay diferencias estadísticamente significativas entre niños y niñas en ninguno de los parámetros. En el resto de los grupos comparados se han obtenido diferencias estadísticamente significativas en algún parámetro: los niños extranjeros tienen más problemas en las relaciones con compañeros $y$, por consiguiente, también en el total de dificultades $(p<0,05)$, los niños cuyos padres tienen un diagnóstico psiquiátrico tienen más problemas emocionales y en las relaciones con los compañeros, lo que obviamente se refleja en el TD. La muestra de pacientes con familias monoparentales es pequeña (21 niños) y existen diferencias significativas también, en problemas emocionales y en las relaciones con los compañeros.

En resumen, los problemas emocionales son más frecuentes en niños cuyos padres tienen algún problema psicológico y en los que conviven con un solo progenitor. Los problemas de relación con iguales son más frecuentes en niños inmigrantes y niños de familia monoparental.

\section{Discusión}

Los niños con problemas psicológicos raramente acuden por su cuenta a pedir ayuda, sino que precisan de los adultos que están a su alrededor para identificar su problema. 
Los pediatras de AP podemos recoger fácilmente la información que proporcionan los padres, que se muestran bien predispuestos a colaborar y el Cuestionario de Capacidades y Dificultades (SDQ) es un instrumento útil. El TD del SDQ permite una clasificación fácil como un primer paso para identificar los problemas psicosociales de los niños en $\mathrm{AP}^{26}$, y ha demostrado ser igual de válido que otros más complejos. En el trabajo se identificó un $5,6 \%$ de niños con un TD anormal y un $10 \%$ con TD en el límite. Este $15 \%$ de niños son susceptibles de estudio, consejo y seguimiento y, si procede, derivación a Salud Mental infantil.

Se ha demostrado que el SDQ realizado por un único informador (padres, en nuestro caso) tiene una especificidad alta $(94 \%)$, pero una sensibilidad baja $(50 \%)^{27}$. Si el resultado del test es normal en todas sus escalas, podemos estar razonablemente tranquilos, los padres predicen bien los niños "sin problemas".

Cuando el resultado es anormal, la probabilidad de que presente un trastorno psiquiátrico es del $50 \%$ y algunos pueden tener problemas sin llegar a cumplir criterios diagnósticos. Esta cifra mejora al incorporar la opinión del profesor hasta un $63 \% \%^{27,28}$.

En un trabajo realizado en $\mathrm{AP}^{29}$ la concordancia de resultados entre SDQ-pa- dres y SDQ-profesores es baja. Ambos identifican a un $20-25 \%$ de niños problemáticos pero no son coincidentes. Los padres identifican mejor los problemas de conducta y los síntomas emocionales y los profesores mejor los problemas de falta de atención y la baja conducta prosocial. Si los pediatras solicitáramos de forma rutinaria informes a los profesores y padres, el número de niños para evaluar sería mucho más elevado que si se pregunta solo a los padres.

En nuestro grupo los problemas de conducta han sido los más comunes, un $10 \%$ y un $10 \%$ adicional en el límite, independientemente de la variable estudiada. Cifras que no se alejan mucho de la prevalencia de 2-16\% publicada para el trastorno oposicionista desafiante en la población infanti ${ }^{30}$ y que coincide con lo expuesto anteriormente sobre los problemas que mejor detectan los padres.

Los problemas de relación con los compañeros se encuentran en $8,4 \%$ y otro $8,4 \%$ en el límite. En este grupo destacan los niños de origen extranjero, es evidente que por la problemática que supone la inmigración en cuanto a desarraigo social y cultural. Son niños de mayor riesgo psicosocial y los pediatras debemos ser sensibles a su situación de mayor fragilidad. También son más frecuentes los problemas de relación en hi- 
jos de familia monoparental o padres con patología psiquiátrica.

Los problemas emocionales se han referido en un $4,5 \%$ de niños y también en este caso afectan más a los hijos de familias monoparentales y con un diagnóstico psiquiátrico. No hemos encontrado diferencias respecto al sexo.

Los síntomas de hiperactividad/falta de atención (TDAH) se encuentran en un $3,9 \%$ de nuestros niños, con otro $11 \%$ en situación límite. No hemos encontrado ninguna diferencia respecto a sexo, país de origen ni composición familiar. Nos ha sorprendido que los síntomas de TDAH hayan sido los cuartos en orden de frecuencia por detrás de los problemas de conducta, problemas de relación y síntomas emocionales. Pensamos que puede explicarse, en parte, porque los padres detectan peor la falta de atención y quizás también porque los puntos de corte españoles son muy altos en esta escala del SDQ, y exige un rango 9-10 para considerarlo anormal (los 5 ítems puntuación máxima).

No se debe olvidar que con el cuestionario SDQ no se obtienen diagnósticos sino que se identifican problemas, que exigen una valoración posterior más completa por el pediatra que deberá decidir la estrategia más oportuna para completar el estudio. No se descarta que un niño con problemas de conducta pueda resultar un hiperactivo o que un niño con síntomas de hiperactividad/falta de atención presente un síndrome de Asperger o trastorno del espectro autista.

El valor del cuestionario SDQ reside en su simplicidad y su reconocida capacidad como instrumento de cribado de la patología psicosocial más frecuente entre la población infantil. Pero tiene limitaciones: no detecta fobias, angustia de separación y trastornos alimentarios.

Debemos ser sensibles a los factores de vulnerabilidad presentes en cada niño. Posiblemente por nuestra formación básicamente organicista tenemos más presentes los factores biológicos como la prematuridad, el bajo peso al nacimiento, Apgar bajo, etc. y muchas veces olvidamos otros muy importantes como los familiares y sociales, de reconocido impacto, como la pobreza o marginalidad. La Guía de Salud Mental para Atención Primaria elaborada por semFYC ${ }^{31}$ dedica un capítulo completo a la monoparentalidad y otro a la patología psiquiátrica familiar, motivos que nos llevaron a recoger estas variables en nuestro estudio. Estos dos parámetros han demostrado ser factores predisponentes para padecer problemas emocionales y de relación con compañeros. 
No se han podido estudiar los datos socioeconómicos por no disponer de ellos.

Los niños de origen extranjero han tenido más problemas de relación, pero no en otras áreas. Parece explicarse por el desconocimiento del idioma, las diferencias culturales y no se puede descartar que existan ciertos prejuicios en la sociedad que les acoge.

La creciente demanda que tenemos los pediatras de orientación y diagnóstico de problemas de comportamiento, falta de atención, dificultades escolares, etc., nos debería hacer reflexionar sobre la necesidad de crear canales de comunicación estables con los servicios educativos. Obtener la información de los profesores puede ser muy importante dada la alta correlación entre dificultades en la escuela con futuros problemas de salud mental y disfunción socia ${ }^{32}$. El profesor conoce al niño, pasa mucho tiempo con él y puede compararle con los demás compañeros.

También es necesario mejorar nuestra capacidad de comunicación con las familias al abordar estos problemas psicosociales, pues es lamentable constatar, como se ha publicado recientemente, que los pediatras no llegamos a comunicar correctamente a las familias el asesoramiento psicológico que pre- tendemos dar. No hay un buen entendimiento, ya que las familias no reconocen haber recibido asesoramiento sobre el problema de salud mental planteado, cuando el pediatra, asegura haberlo dado ${ }^{33}$.

\section{Conclusión}

El cribado rutinario sobre la salud mental infantil con el SDQ es factible en AP. No precisa inversión tecnológica, sino unos minutos para la corrección del cuestionario por los profesionales que atienden al niño. La información ofrecida por los padres es útil pero infraidentifica los problemas, que podrían descubrirse si también se dispusiera de la valoración del profesor. No obstante, es una oportunidad para que afloren algunos problemas que de otra manera no se hubieran planteado. La historia sociofamiliar debe investigarse, pues incide claramente en la salud mental del niño.

Se necesita algún algoritmo que nos ayude a decidir cuándo debemos solicitar información del profesor y cómo interpretar los resultados no concordantes entre padres y profesores. Establecer un canal de comunicación reglado con los profesionales de la educación mejoraría la atención que prestamos a nuestra población infantil. 


\section{Agradecimientos}

Queremos agradecer la colaboración en la distribución de los cuestionarios y en la recogida de datos a las enfermeras Belinda Escrig García y Sara Folch Manuel.

\section{Bibliografía}

1. Arroba Basanta ML. Introducción. En: AMPap, ed. II Curso Primavera 2007. Madrid: Exlibris Ediciones; 2007. p. 7-8.

2. Observatorio de Salud Infantil y Perinatal. (OSIP). Dirección General de Salud Pública de la Conselleria de Sanitat de la Comunidad Valenciana; 2009 [consultado el 04/06/2009]. Disponible en www.sp.san.gva.es/DgspWeb/sscc/opciones2. jsp?Opcion=SANMS119\&CodPunto $=2247 \& M e$ nuSup=SANMS\&Nivel $=2$

3. Narbona J. Alta prevalencia del TDAH: ¿niños trastornados o sociedad maltrecha? Rev Neurol. 2001;32(3):229-31.

4. Gómez de Terreros I. Violencia y juventud. An Pediatr (Barc). 2009;70(3):205-8.

5. Puig de la Bellacasa J, Carrasco $E$, Languin $A$, Mora E. Atención a niños en situación de riesgo psicosocial. Experiencia de un centro de salud. Rev Pediatr Aten Primaria. 1999;1:57-68.

6. Boydell B, Bowers S. Estimating the prevalence of early childhood serious emotional/behavioral disorders: challenges and recommendations. Public Health Reports. 2006;121:303-10.

7. Avellanosa Caro I. Salud mental infantojuvenil en España. Rev Pediatr Aten Primaria. 1999;1: 119-27.

8. Pedreira Massa JL, Sardinero E. Prevalencia de trastornos mentales de la infancia en Atención Primaria pediátrica. Actas Luso-Esp Neurol Psiquiatr. 1996;24(4):173-90.

9. Rodríguez Hernández PJ. Estudio de la prevalencia de los trastornos mentales infantiles en la
Comunidad Autónoma Canaria (tesis doctoral). Tenerife: Universidad de La Laguna; 2006.

10. Tizón García JL. Prevención e intervención en la salud mental de la primera infancia desde los dispositivos de Atención Primaria. Rev Pediatr Aten Primaria. 2002;4(13):81-106.

11. Martínez González C. Los problemas de salud mental. Un reto para el pediatra. Rev Pediatr Aten Primaria. 2002;4(13):139-44.

12. Cassidy LI, Jellinek MS. Approaches to recognition and management of childhood psychiatric disoders in pediatric primary care. Clin Pediatr North Am. 1998;45:1037-52.

13. Williams J, Klinepeter K, Palmes G, Pulley A, Foy JM. Diagnosis and treatment of behavioral health disorders in Pediatric Practice. Pediatrics. 2004;114(3):601-6.

14. Romero Escobar $H$, Naenen Hernani $K$, Martínez Manrique I, Cantó Diez T, Canals Baeza A. Propuesta de inclusión de una escala de detección del TDAH en el programa del niño sano en Atención Primaria. Acta Pediatr Esp. 2006;64(3):99102.

15. Borowsky IW, Mozayeny S, Ireland M. Brief psychosocial screening at health supervision and acute care visits. Pediatrics. 2003;112:129-33.

16. Goodman R. The Strengths and Difficulties Questionnaire: a research note. J Child Psychology Psychiatry. 1997;38:581-6.

17. Vostanis P. Strengths and Difficulties Questionnaire: research and clinical applications. Curr Opin Psychiatry. 2006;19(4):367-72.

18. Hawes DJ, Dadds MR. Australian data and psychometric properties of the Strengths and Diffi- 
culties Questionnaire. Australian and New Zealand J Psychiatry. 2004;38:644-51.

19. Malmberg $M$, Rydell AM, Smedje H. Validity of the Swedish version of the Strengths and Difficulties Questionnaire (SDQ-Swe). Nord J Psychiatry. 2003;57(5):357-63.

20. Koskelainen $M$, Sourander $A$, Kaljonen A. The Strengths and Difficulties Questionnaire among finnish school aged children and adolescents. Eur Child Adolesc Psychiatry. 2000;9(4): 277-84.

21. Woerner W, Becker A, Rothenberger A. Normative data and scale properties of the German parent SDQ. Eur Child Adolesc Psychiatry. 2004;13 Suppl 2:s3-s10.

22. Van Widenfelt BM, Goedhart AW, Treffers PDA, Goodman R. Dutch version of the Strengths and Difficulties Questionnaire (SDQ). Eur Child Adolesc Psychiatry. 2003;12:281-9.

23. Bourdon KH, Goodman R, Rae DS, Simpson G, Koretz DS. The Strengths and Difficulties Questionnaire: US. Normative data and psychometric properties. J Am Acad Child Adolesc Psychiatry. 2005;44:557-64.

24. Rodríguez Hernández PJ, Herreros O. Historia clínica, evaluación y diagnóstico en Psiquiatría Infantil. Curso de Formación Continuada en Psiquiatría Infantil. 2005 [consultado el 04/06/2009]. Disponible en www.sepeap.org/index.php?me nu=416

25. Rodríguez Hernández PJ. Trastornos psiquiátricos infantiles de etiología neurobiológica y su despistaje en Atención Primaria. BSCP Can Pediatr. 2006;30:9-18.
26. Crone MR, Vogels AG, Hoekstra F, Treffers $P$, Reijneveld $S$. A comparison of four scoring methods based on the parent-rated SDQ as used in the Dutch Preventive Child Health Care System. BMC Public Health. 2008;8:106.

27. Goodman R, Ford T, Simmons H, Gatward $\mathrm{R}$, Meltzer $\mathrm{H}$. Using the Strengths and Difficulties Questionnaire (SDQ) to screen for child psychiatric disorders in a community sample. Br J Psychiatry. 2000;177:534-9.

28. Ford T, Sayal K, Meltzer H, Goodman R. Parental concerns about their child's emotions and behaviour and referral to specialist services: general population survey. BMJ. 2005;331:1435-6.

29. Brown JD, Wissow LS, Zachary C, Bartlett E, Horn I. Parent and teacher mental health ratings of children using primare care services: inter-rater agreement and implications for mental health screenig. Ambul Pediatr. 2006;6(6):347-51.

30. Rigau-Ratera E, García-Nonell C, ArtigasPallares J. Tratamiento del trastorno de oposición desafiante. Rev Neurol. 2006;42 Supl 2:s83-s88.

31. Guía de Salud Mental en Atención Primaria. Barcelona: semFYC; 2001.

32. De Socio J, Hootman J. Children's mental health and school success. J Sch Nurs. 2004;20: 189-96.

33. Brown JD, Wissow LS. Disagreement in parent and primary care provider reports of mental health counseling. Pediatrics (Ed esp). 2008;66(6): 343-50. 